\title{
Synthesis and Properties of a Heteroleptic $\mu$-Nitrido Tetrapyrrolic Complex
}

\author{
Gloria Zanotti, Giuseppe Mattioli, Sara Notarantonio, Anna Maria Paoletti, \\ Gentilina Rossi, Aldo Amore Bonapasta, and Giovanna Pennesi ${ }^{\circledR}$
}

\author{
Dedicated to Prof. Claudio Ercolani - pioneer in Phthalocyanines Chemistry \\ on the occasion of his $75^{\text {th }}$ birthday
}

\author{
CNR, Istituto Struttura della Materia, Via Salaria Km. 29.3, P.B. 10, 00015 Monterotondo Stazione, Rome, Italy \\ ${ }^{\circledR}$ Corresponding authorE-mail: gianna.pennesi@ism.cnr.it
}

A novel $\mu$-nitrido iron phthalocyanine-iron porphyrin complex has been synthesised and characterised, preliminary DFT calculations have been performed in order to elucidate its UV-vis spectrum.

Keywords: Synthesis, phthalocyanine, porphyrin, $\mu$-nitrido dimer.

Multi-component organic chromophores having intense visible absorption characteristics covering large part of solar radiation are currently an object of much attention in view of their exploitation as artificial light-harvesting antenna.

In particular those containing the phthalocyanine $(\mathrm{Pc})$ and porphyrin $(\mathrm{P})$ macrocyclic ligands have been recently studied being interesting for the peculiar photoinduced energy transfer processes ${ }^{[1]}$ and being also attractive for their potential use in the field of molecular photovoltaics, artificial photosynthesis and molecular electronic applications. ${ }^{[2-4]}$

Several strategies can be used to link each other the two different subunits to obtain a hetero phthalocyanine-porphyrin dyads, resulting in different geometries and properties. It is possible for example to link the different moieties through a conjugated or not spacer between the peripheral positions of one macrocycle ${ }^{[5]}$ toward either the periphery or the central metal of the other macrocycle. ${ }^{[1]}$ Moreover single atom $(\mathrm{O}$, $\mathrm{N}, \mathrm{C}$ ) bridged systems as $\mu$-oxo, $\mu$-nitrido and $\mu$-carbido can also allow a combination, via metal centres, leading to homo/heterometallic and homo/heteroleptic systems. ${ }^{[6]}$ To the aim recalled above, it should be important that the orbital interactions could promote an electronic communication along the stack thereby optical absorption will change and, in the case of complementary chromophores, extend.

As other analogues mixed-ligand porphyrin/phthalocyanine bridged systems are expected to mutate the electronic configuration and to extend the visible absorption to the near IR wavelength.

Among the $\mu$-nitrido species, the mixed-ligand tetraphenylporphyrin-phthalocyanine $\mu$-nitrido complex of iron was synthesised and fully characterised as solid material by Ercolani et al. $;^{[7,8]}$ it showed a good thermal stability ( $>$ $300^{\circ} \mathrm{C}$ ) and an interesting electronic and magnetic exchange between the iron centres trough the linear $\mathrm{Fe}-\mathrm{N}-\mathrm{Fe}$ bond system. Unfortunately its optical (UV-vis) spectrum was not reported likely because of its very scarce solubility in non-donor solvent. Being this feature indispensable for the processability of these material in view of technological applications, we attempted to increase the solubility of such species and we present here preliminary investigations on the substituted analogous ( $\mu$-nitrido)[((tetraphenylporphyrinato) iron)-(tetra-tert-butylphthalocyaninato)iron], I. ${ }^{\S}$

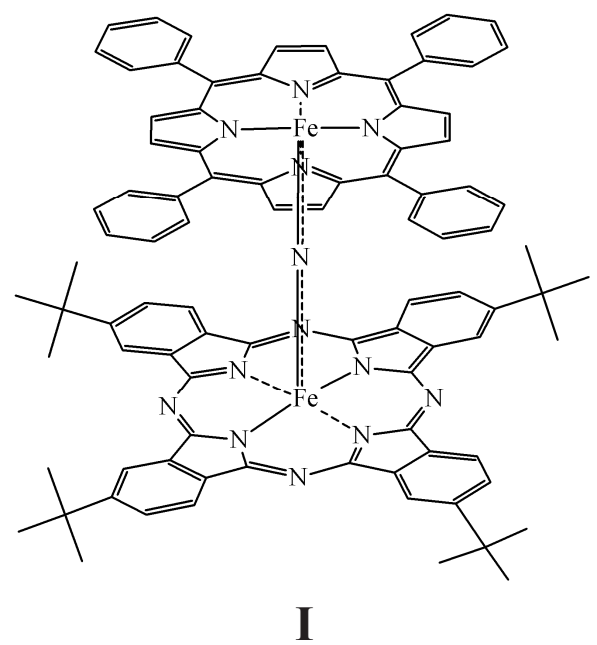

${ }^{\S}$ All reagents were obtained from commercial source, Aldrich, Fluka or CarloErba and used after proper purification. All solvents were anhydrous and distilled before use. Reactions were monitored by Thin Layer Chromatography (TLC) employing a polyester layer coated with $250 \mathrm{~mm}$ F254 silica gel. The purification of compounds was performed by column chromatography using silica gel CarloErba Reactifs SDS 60A C.C. $35-70 \mathrm{~mm}$. The crude was dissolved in pyridine and the desired product was purified by column chromatography on silica gel using first dichloromethane and then a mixture of dichloromethane-ethyl acetate 1:1 as mobile phase. Infrared spectra were recorded on a Shimadzu IR prestige-21 spectrometer in $\mathrm{KBr}$ pellets or in nujol mull. UVvis spectra were recorded in solution on a Perkin-Elmer Lambda 950 UV-vis/NIR spectrophotometer. The mass spectra have been recorded at Toscana Life Science facility in a MALDI-TOF/ TOF Ultraflex III (Bruker) by MALDI-TOF method ( $\alpha$-cyano-4hydroxycinnamic acid (HCCA) as matrix). DFT calculations have been performed at an LSD-GGA+U level, ${ }^{[14]}$ in a supercell approach, by using atomic pseudopotentials and a plane wave basis set. 
The compound I was synthesised mixing equivalent amounts of iron(III) tetraphenylporphyrin azide $\left(\left(\mathrm{N}_{3}\right)\right.$ FeTPP) and iron(II) tetra-tert-butylphthalocyanine (FeTTBPc) in freshly distilled xylene, stirring the solution at $120^{\circ} \mathrm{C}$ for 8 hours under nitrogen atmosphere. The resulting solution was then concentrated and the desired product was obtained as the pyridine adduct after separation with column chromatography. Thermogravimetric analysis showed a weight loss of $11 \%$ at $150-160{ }^{\circ} \mathrm{C}$, and this is consistent with the calculated loss of $10.50 \%$ for 2 molecules of pyridine.

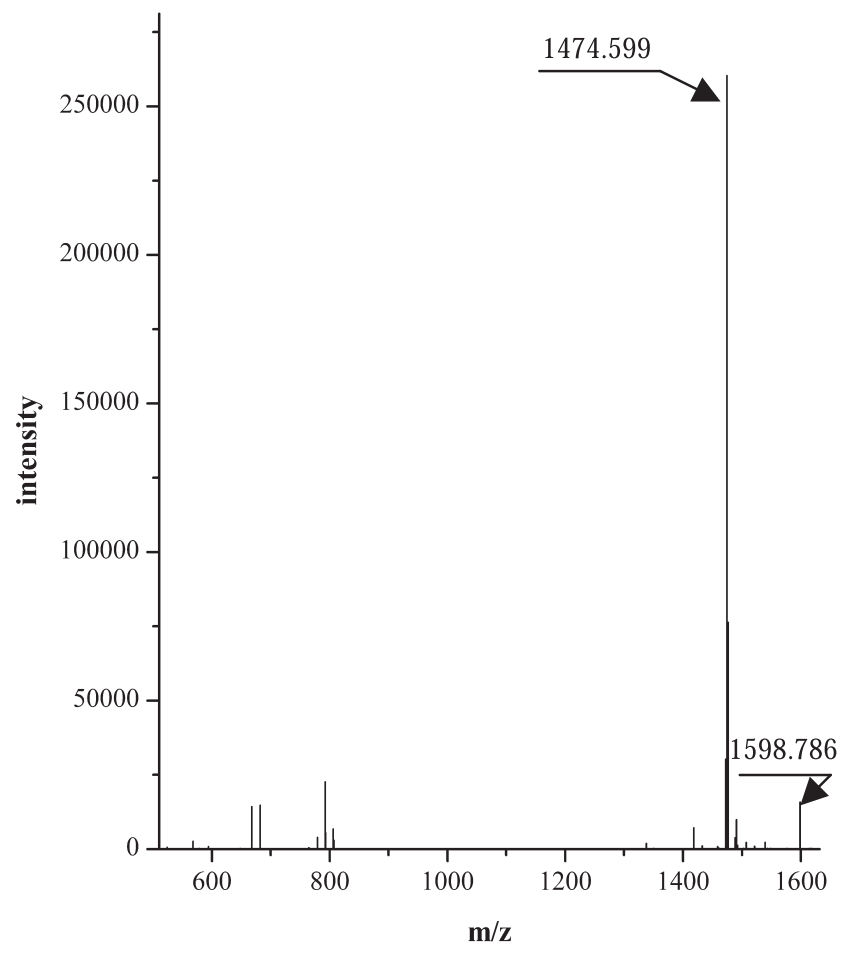

Figure 1. MALDI-TOF mass spectrum of compound $\mathbf{I}$.

The complex was characterised by elemental analysis $\left(\mathrm{C}_{92} \mathrm{H}_{76} \mathrm{~N}_{13} \mathrm{Fe}_{2}\right.$ (1475.37), calcd. C 74.90, H 5.19, N 12.34; found $\mathrm{C} 71.30, \mathrm{H} 4.89, \mathrm{~N} 11.60)$ and MALDI-TOF mass spectrometry $\left(\mathrm{m} / \mathrm{z}=\mathrm{M}^{+} 1474.599\right)$. The presence of small amounts of ( $\mu$-nitrido) iron tetra-tert-butyl phthalocyanine as contaminant could not always be avoided and scrupulous care must be taken for its minimisation both in reaction conditions and in chromatographic separation. In fact, besides the expected fragmentation referable to both the moieties of the system, in the MALDI spectrum (Figure 1) it is present also the peak assignable to the above reported contaminant $(\mathrm{m} / \mathrm{z}=1598)$.

The infrared spectrum showed an intense absorption peak at $927 \mathrm{~cm}^{-1}$ attributable to the $v_{\text {as }}(\mathrm{Fe}-\mathrm{N}-\mathrm{Fe})$ group; the same stretching absorption in the corresponding homoleptic bridged systems, i.e. [(TPP)Fe-N-Fe(TPP)] $]^{[9]}$ and [TTBPcFe$\mathrm{N}-\mathrm{FeTTBPc}]^{[10]}$ is displayed at 910 and 938 respectively and very close to the unsubstituted analogue. ${ }^{[7]}$ This band disappears when compound $\mathbf{I}$ is adducted by $N$-bases and can be detected only after their elimination.

The compound appears stable in air when it is adducted and it is soluble in non donor solvents such as dichloromethane or chloroform and in coordinating solvents as pyridine or tetrahydrofuran. The normalised UV-visible spectrum of I in pyridine, together with those of the precursors in the same solvent, is shown in Figure 2. It appears indeed very similar to the one reported for hetero-bimetallic $\mu$-nitrido system (TPP)Mn-N-Fe(Pc). ${ }^{[1]}$ Also for compound I, as clearly visible, the spectrum is not a mere sum of the precursors' spectra: in the range between 300 and $500 \mathrm{~nm}$ the peaks of Soret bands of porphyrin and phthalocyanine are substantially retained, but for this latter a slight red-shift is observed. The rest of the spectral range presents a broad envelope resulting from the combination of $Q$ bands of the complementary chromophores that extend the absorption up to the near infrared. These interesting features motivate to further experimental and theoretical investigations being this kind of studies relevant for a deep comprehension of the electronic interactions in these systems as pointed out in a recent paper. $^{[12]}$ In particular the nature of the absorption at $742 \mathrm{~nm}$ needs to be elucidated, whether it is attributable to a relevant red-shift of the $Q$ band of the phthalocyanine or it is a new band arising from transition between new states of the dyad.

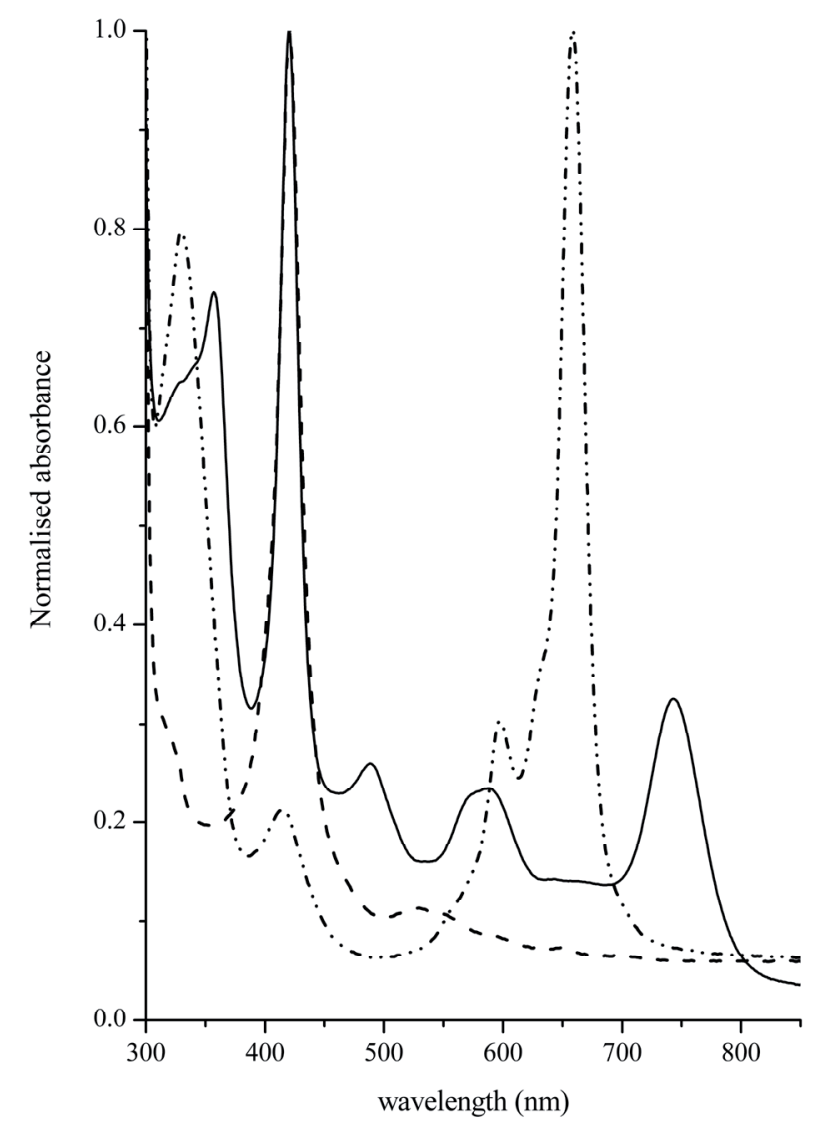

Figure 2. Normalised UV-visible spectra of compound I (solid line); iron tetra-tert-butyl phthalocyanine (dot-dash line); iron tetraphenylporphyrin azide (dash line).

In this regard, preliminary indications have been given by ab initio DFT (Density Functional Theory) calculations, in a theoretical framework already applied in previous studies of similar molecular systems ${ }^{[13]}$. The geometries of the compound $\mathbf{I}$ as well as of models of its components (i.e., iron tetra-tert-butylphthalocyanine, FeTTBPc, and iron tetraphenyl-porphyrin chloride, Cl-FeTPP) have been optimized by fully relaxing the atomic forces. Then, the evolution of the relative positions of the frontiers HOMO-LUMO when 


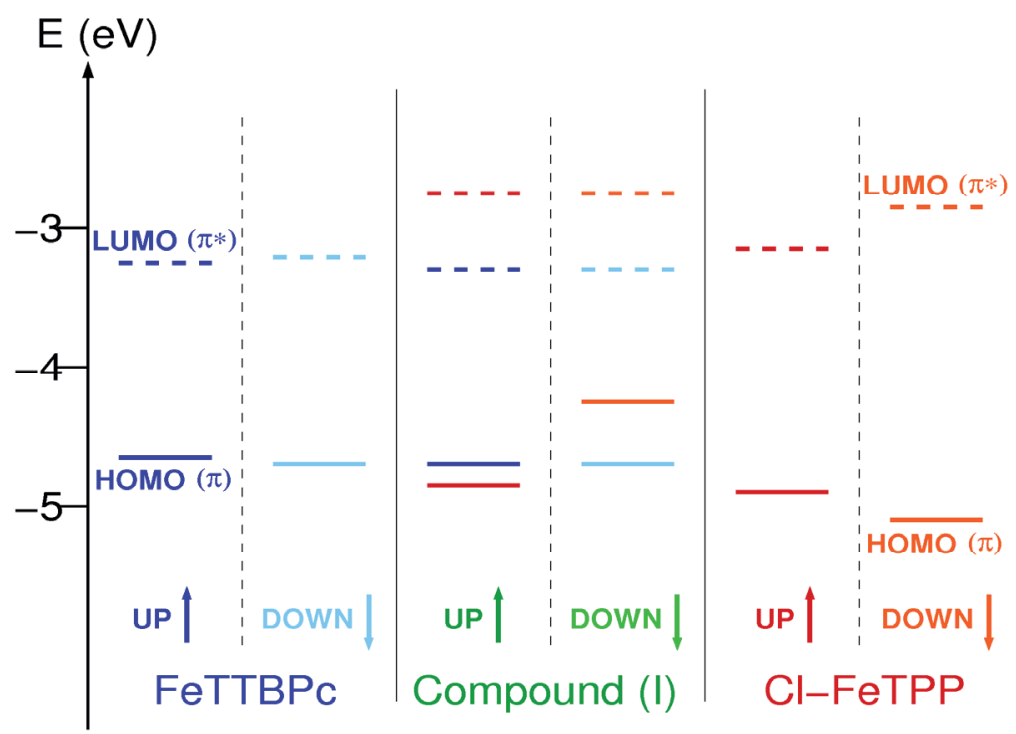

Figure 3. Schematic electronic level diagram showing the relative positions of the $\operatorname{HOMO}(\pi)$ and $\operatorname{LUMO}\left(\pi^{*}\right)$ for (from left side to right side), the FeTTBPc, compound I, and Cl-FeTPP molecules. Spin up and spin down levels are separately shown in the figure. For the sake of clearness, in the case of compound $\mathbf{I}$, the electronic levels induced by the two components are indicated with different colours.

the two components are combined in I can be considered by looking at the electronic level diagram sketched in Figure 3. Although these results represent only a first step towards a theoretical investigation of the electronic properties of $\mathbf{I}$, a first and very qualitative analysis of that diagram shows that the difference in energy between the HOMO-LUMO states corresponding to the Pc macrocycle change from 1.7 $\mathrm{eV}$ and $1.7 \mathrm{eV}$ (up and down spin channels, respectively) in the FeTTBPc to the values of $1.7 \mathrm{eV}$ and $1.4 \mathrm{eV}$ (in the corresponding spin channels) in the compound I, thus suggesting a red-shift of the phthalocyanine $Q$ band.

Acknowledgements. Gentilina Rossi, Giovanna Pennesi and Anna Maria Paoletti would like to thank Prof. C.Ercolani for his longstanding collaboration in the field and for having transmitted them determination, highly professionality, constancy and enthusiasm in the research job.

\section{References}

1. Maligaspe E., Kumpulainen T., Lemmetyinen H., Tkachenko N.V., Subbaiyan N.K., Zandler M.E., D'Souza F. J. Phys. Chem. A 2010, 114, 268-277.

2. Walter M.G., Rudine A.B., Wamser C.C. J. Porphyrins Phthalocyanines 2010, 14, 759-792.
3. Rio Y., Vazquez P., Palomares E. J. Porphyrins Phthalocyanines 2009, 13, 645-651.

4. Rio Y., Rodriguez-Morgade M.S., Torres T. Org. Biomol. Chem. 2008, 6, 1877-1894.

5. Fortage J., Göransson E., Blart E., Becker H.C., Hammarström L., Odobel F. Chem. Commun. 2007, 4629-4631.

6. a) Floris B., Donzello M.P., Ercolani C. In: The Porphyrin Handbook (Kadish K.M., Smith K.M., Guilard R., Eds.). Academic Press: San Diego, 2003, Vol. 18, p 1. b) Zhao Z., Cammidge A.N., Hughes D.L., Cook M. J. Org. Lett. 2010, 12, 5138-5141.

7. Ercolani C., Hewage S., Heucher R., Rossi G. Inorg. Chem. 1993, 32, 2975-2977.

8. Ercolani C., Jubb J., Pennesi G., Russo U., Trigiante G. Inorg. Chem. 1995, 34, 2535-2541.

9. Summerville D.A., Cohen I.A. J. Am. Chem. Soc. 1976, 98, 1747-1755.

10. Sorokin A.B., Kudrik E.V., Bouchu D. Chem. Commun. 2008, 2562-2564.

11. Donzello M.P., Ercolani C., Kadish K.M., Zhongping O., Russo U. Inorg. Chem. 1998, 37, 3682-3688.

12. Silaghi-Dumitrescu R., Makarov S.V., Uta M., Dereven'kov I.A., Stuzhin P.A. New J. Chem. 2011, 35, 1140-1145.

13. Zanotti G., Angelini N., Paoletti A.M., Pennesi G., Rossi G., Amore Bonapasta A., Mattioli G., Di Carlo A., Brown T. M., Lembo A., Reale A. Dalton Trans. 2011, 40, 38-40.

14. Giannozzi, P., et al., J. Phys.: Condens. Matter. 2009, 21 , 395502. 